\title{
Questes
}

Revue pluridisciplinaire d'études médiévales

\section{Ce qui circule : mots et cadeaux entre échange et rejet dans l'Orient des croisades}

Florian Besson

\section{(2) OpenEdition}

Édition électronique

URL : http://journals.openedition.org/questes/4397

DOI : 10.4000/questes.4397

ISSN : 2109-9472

Éditeur

Les Amis de Questes

\section{Édition imprimée}

Date de publication : 31 mars 2017

Pagination : 81-98

ISSN : 2102-7188

\section{Référence électronique}

Florian Besson, «Ce qui circule : mots et cadeaux entre échange et rejet dans l'Orient des croisades », Questes [En ligne], 35 | 2017, mis en ligne le 15 avril 2017, consulté le 03 mai 2019. URL : http:// journals.openedition.org/questes/4397; DOI : 10.4000/questes.4397 


\title{
Ce qui circule : mots et cadeaux entre échange et rejet dans l'Orient des croisades ${ }^{1}$
}

\author{
Florian BESSON
}

Université Paris-Sorbonne

Depuis une vingtaine d'années, les recherches soulignent, contre l'image avancée par Joshua Prawer d'une société d'apartheid ${ }^{2}$, l'importance des rencontres, des échanges et des contacts entre Latins, musulmans, Grecs et chrétiens d'Orient ${ }^{3}$ dans les États latins fondés après le succès de la première croisade. Ces rencontres ont pu prendre plusieurs formes: transferts culturels, notamment sur le plan de l'industrie sucrière $^{4}$, des techniques de construction ${ }^{5}$, ou du savoir médical ${ }^{6}$;

\footnotetext{
${ }^{1}$ Cet article doit énormément à Pauline Guéna et Catherine Kikuchi, que je remercie.

${ }^{2}$ Voir par exemple Joshua Prawer, «Colonization activities in the latin kingdom of Jerusalem », Revue belge de philologie et d'histoire, 1951, vol. 29, n 4, p. 10631118.

${ }^{3}$ Par «Latins », on entendra tous les fidèles de la chrétienté romaine, qu'ils soient originaires de l'Occident ou nés en Orient ; par «Grecs », les sujets de l'empire byzantin. Ces appellations sont en elles-mêmes trompeuses, car elles simplifient abusivement une situation bien plus complexe : rappelons que les musulmans sont divisés en plusieurs courants - sunnites, shiites, ismaéliens, Druzes - tout comme les Chrétiens d'Orient - Melkites, Arméniens, Nestoriens, Jacobites, etc. Il faut donc entendre ces ethnonymes comme des outils opératoires et non des concepts.

${ }^{4}$ Voir William D. Phillips, "Sugar production and trade in the Mediterranean at the time of the crusades », dans The Meeting of two worlds: cultural exchange between east and west during the period of the crusades, dir. Vladimir P. Goss et Christine V. Bornstein, Kalamazoo, Medieval Institute Publications, coll. «Studies in Medieval Culture », 1986, p. 393-406 ; Mohamed Ouerfelli, Le Sucre : production, commercialisation et usages dans la Méditerranée médiévale, Leiden, J. Brill, coll. «Medieval Mediterranean », 2008.

${ }^{5}$ Nicolas Prouteau, «L'Art de la charpenterie et du génie militaire dans le contexte des croisades, recrutement et fonctions des techniciens francs (XII ${ }^{\mathrm{e}}$-XIII ${ }^{\mathrm{e}}$ siècles) », dans La Fortification au temps des croisades, dir. Nicolas Faucherre, Jean Mesqui et
} 
échanges linguistiques, les Latins ramenant les mots «douane», « gabelle», « amiral », et les Arabes prenant les termes «bourgeoisie», «anguille ${ }^{7} »,\left\langle\operatorname{vassal}^{8} » ;\right.$ rencontres individuelles, enfin, marquées du coin des amitiés, des mariages entre communautés, des acculturations. Le tout, rappelons-le afin de ne pas tomber dans une vision trop irénique, sur fond de guerre, de conflit, d'opposition religieuse, politique et ethnique. Il y a quelques mois, dans le séminaire de Questes sur le thème «Faire communauté », j'avais proposé de repenser l'ensemble de ces rapports en termes de middle ground, concept avancé par Richard White ${ }^{9}$, c'est-à-dire de souligner que tous ces échanges et ces contacts participaient de la création d'un monde commun, au même titre que les incompréhensions et les malentendus ${ }^{10}$. Ce qui se crée, c'est une société complexe, dans laquelle les identités confessionnelles et ethniques existent bel et bien et structurent les appartenances sociales, mais où les trajets personnels se font plus riches et plus fluides. Cette fluidité est d'ailleurs, jusqu'à un

Nicolas Prouteau, Rennes, Presses Universitaires de Rennes, coll. « Archéologie et culture », 2004, p. 279-286.

${ }^{6}$ Émilie Savage-Smith, «New evidence for the frankish study of arabic medical texts in the crusader period », Crusades, 2006, $\mathrm{n}^{\circ} 5$, p. 99-112 ; Jonathan Rubin, "The Use of the "Jericho Tyrus" in theriac: a case study in the history of the exchanges of medical knowledge between western Europe and the realm of Islam in the Middle Ages », Medium Aevum, 2014, LXXXIII, n 2, p. 234-253.

${ }^{7}$ Balazs Major, "Anklîs". A possible trace of european presence in the medieval syrian vocabulary », dans More modoque : die Wurzeln der europäischen Kultur und deren Rezeption im Orient und Okzident. Festschrift für Miklos Maroth zum siebzigsten Geburtstag, dir. Pal Fodor, Gyula Mayer, Martina Monostori, Budapest, Forschungzentrum für Humanwissenschaften der Ungarischen Akademie der wissenchaften, 2013, p. 377-384.

${ }^{8}$ Moshe Sharon, «Vassal and fasal : the evidence of the farkah inscription from 608/1208 », Crusades, 2005, n 4, p. 117-130.

${ }^{9}$ Richard White, Le Middle Ground. Indiens, Empires et Républiques dans la région des Grands Lacs, 1650-1815, Toulouse, Anacharsis, coll. «Essais », 2009.

${ }^{10}$ Florian Besson, «Vivre en communauté ou entre communautés? Une réflexion sur le middle ground des États latins d'Orient », Questes, n 32, Faire communauté, numéro coordonné par Julia Conesa Soriano et Julie Pilorget, 2016, p. 35-50. 
certain point, une caractéristique de ces sociétés de frontière marquées par les contacts entre cultures et entre religions ${ }^{11}$.

Les exemples sont nombreux qui illustrent l'impossibilité de placer les acteurs dans des cases précises ou dans une grille dont la religion serait l'abscisse et l'ethnicité l'ordonnée. Ainsi du Livre au Roi, texte juridique rédigé dans les premières années du XIII ${ }^{\mathrm{e}}$ siècle, qui autorise un chevalier franc à servir dans une armée musulmane s'il a obtenu le congé du roi auparavant ${ }^{12}$; ou de Hamdān al-Atharibī, un musulman qui tient un fief dans le comté de Tripoli et sert de messager et d'ambassadeur à la fois pour son suzerain franc, pour les émirs de Damas et d'Alep, et pour les Nizârites du Liban, aussi appelés les Assassins ${ }^{13}$.

Ces exemples dessinent un paysage complexe, qui sera mieux saisi si on abandonne l'idée, manichéenne et réductrice, d'un choc entre deux «mondes », quand ce n'est pas entre «civilisations». Le concept de Richard White a l'avantage de mettre l'accent sur la création, donc sur la fécondité des interactions et des rapports. C'est ce point que je vais reprendre et approfondir, en m'intéressant spécifiquement à ce qui s'échange dans les rencontres, non pas entre cultures, mais entre individus. Je me concentrerai dans cet article sur les rapports politicodiplomatiques, en excluant, notamment, toutes les rencontres

${ }^{11}$ Entre Islam et chrétienté. La territorialisation des frontières, XI $I^{e}$ XVI $I^{e}$ siècles, dir. Stéphane Boissellier et Isabel Cristina Ferreira Fernandes, Rennes, Presses Universitaires de Rennes, coll. «Histoire», 2015; Johannes Preiser-Kapeller, «Liquid frontiers : a relational analysis of maritime Asia Minor as a religious contact zone in the thirteenth-fifteenth centuries », dans Islam and Christianity in Medieval Anatolia, dir. A. Peacock, Bruno De Nicola, Sara Nur Yildiz, Burlington, Ashgate, 2015, p. 117-145.

${ }^{12}$ Le Livre au roi, éd. Myriam Greilsammer, Paris, Académie des Inscriptions et Belles-Lettres, coll. «Documents relatifs à l'histoire des croisades », 1995, cit. chap. 22, p. 200-201.

${ }^{13}$ Benjamin Z. Kedar, «Some new sources on palestinian Muslims before and during the crusades », dans Die Kreuzfahrerstaaten als multikulturelle Gesellschaft, dir. Hans E. Mayer, München, Oldenbourg, coll. «Schriften des Historischen Kollegs », 1996, p. 129-140. 
commerciales, abondamment documentées et étudiées pour toute l'époque $^{14}$; faute de place, je laisse également de côté la question, importante, des circulations de personnes : prisonniers et otages font en effet partie intégrante des échanges diplomatiques ${ }^{15}$. Ainsi, qu'est-ce qui circule, et qu'est-ce qui bloque, lorsque se rencontrent un Latin et un musulman, ou un Latin et un Grec ?

\section{Des mots}

\section{Parler pour s'entendre}

Les mots sont la première chose qui circule. On connaît l'importance de l'oralité dans les pratiques sociales médiévales, en Orient comme en Occident ${ }^{16}$, et en particulier dans les pratiques politiques de l'aristocratie féodale, avec l'importance clé du serment et le rôle majeur joué par le consilium. Comme l'écrivent Jacques Godbout et Alain Caillé dans leur analyse du don, la parole est la première chose que l'on donne pour créer la confiance, et « la première fonction de la parole est d'abord de circuler, d'être donnée et rendue, d'aller et de $\operatorname{venir}^{17}{ }^{17}$. Quand deux personnes se rencontrent, elles parlent. L'impossibilité de parler joue à l'inverse comme le symbole même de la non-rencontre, et donc de la division. Foucher de Chartres indique ainsi que l'armée de la première

\footnotetext{
${ }^{14}$ Pour une approche historiographique des enjeux du commerce entre cultures, voir Francesca Trivellato, «The Historical and Comparative Study of Cross-cultural Trade », dans Religion and Trade. Cross-cultural Exchanges in World History, 10001900, dir. Catia Antunes, Leor Halevi, Francesca Trivellato, Oxford, Oxford University Press, 2014, p. 2-25.

${ }^{15}$ Voir, sur ce sujet, Yvonne Friedman, Encounter between enemies : captivity and ransom in the Latin Kingdom of Jerusalem, Leiden, Brill, coll. «Cultures, Beliefs and Traditions », 2002.

${ }^{16}$ Oralité et lien social au Moyen Âge (Occident, Byzance, Islam). Parole donnée, foi jurée, serment, dir. Marie-France Auzépy et Guillaume Saint-Guillain, Paris, ACHCByz, coll. « Monographies », 2008.

${ }^{17}$ Jacques T. Godbout et Alain Caillé, L'Esprit du don, Paris, La Découverte, coll. «Textes à l'appui. Série Anthropologique », 1992, p. 22.
} 
croisade est composée de tant de peuples différents qu'il lui était impossible de répondre à tous, faute de maîtriser toutes les langues ${ }^{18}$.

La pluralité linguistique joue alors comme un écho de la Tour de Babel, immédiatement annulée par l'union de tous dans la croisade, qui n'en apparaît que plus miraculeuse. On retrouve le même schéma chez Eudes de Deuil, racontant la deuxième croisade : devant Constantinople, les Français et les Allemands en viennent aux mains, et « il en résulta une rixe, avec des clameurs épouvantables ; car les uns n'entendant pas les autres, chacun criait à tue-tête, et parlait sans se faire comprendre ${ }^{19} »$. Littéralement, on ne s'entend pas, et l'existence même de cette expression renvoie à la place cruciale de l'échange de mots dans le lien social.

On observe cet aspect partout dans les sources, et cela se manifeste, notamment, par le rôle clé des ambassadeurs, des messagers, des traducteurs, bref de tous les intermédiaires, ces go-betweens ${ }^{20}$ qui, par leurs compétences diplomatiques et linguistiques, savent tisser des liens et ouvrir des portes ${ }^{21}$. Ces derniers savent souvent profiter de leurs talents

\footnotetext{
${ }^{18}$ Foucher de Chartres, Historia Iherosololymitana. Gesta Francorum Iherusalem Peregrinantium, dans Recueil des Historiens des Croisades (désormais abrégé en RHC), Historiens Occidentaux, tome III, Paris, Imprimerie impériale, 1866, p. 311485, cit. chap. XIII, p. 336-337 : «Sed quis unquam audivit tot tribus linguae in uno exercitu, cum ibi adessent Franci, Flandri, Frisi, Galli, Britoni, Allobroges, Lotharingi, Alemanni, Bajoarii, Northmanni, Scoti, Anglici, Aquitani, Itali, Apuli, Iberi, Daci, Graeci, Armenii? Quod si vellet me alloqui Britannus, vel Teutonicus, neutro respondere saperem ».

${ }^{19}$ Eudes de Deuil, La Croisade de Louis VII, roi de France, Paris, P. Geuthner, coll. «Documents relatifs à l'histoire des croisades», 1949, cit. chap. III, p. 36 : «Inde rixa, imo garritus oboritur, ubi enim alter alterum non intelligens, cum clamosa voce impetit, garritus est $\gg$. Je traduis.

${ }^{20} \mathrm{Au}$ sens, par exemple, de Andreas Höfele et Werner von Koppenfels (éd.), Renaissance go-betweens. Cultural exchange in early modern Europe, Berlin, Walter de Gruyter, 2005.

${ }^{21}$ Pour l'Orient latin, voir, malgré plusieurs erreurs ou naïvetés, Katrine A. Tuley, " "For We Who Were Occidentals Have Become Orientals": the Evolution of Intermediaries in the Latin East, 1095-1291 », Honors Research Thesis, Ohio State University, sous la direction de Sarah-Grace Keller, 2012. Pour une réflexion plus globale sur la place des intermédiaires, on lira François-Xavier Fauvelle-Aymar, Le Rhinocéros d'or. Histoires du Moyen Âge africain, Paris, Alma, 2013.
} 
pour obtenir des avantages matériels: position sociale, richesse, protection lors des sièges. C'est le cas notamment des Banū Munqidh, seigneurs de Shayzar, sur l'Oronte ${ }^{22}$, qui sont décrits comme « de nobles natures, aimant tout le monde, et, dans toutes les occasions, de bons médiateurs $^{23}{ }^{2}$. Cela dit, Daniel König a très justement souligné qu'il y avait souvent un prix à payer pour être ainsi placé entre les cultures, ne serait-ce qu'en termes de malaise social et mental ${ }^{24}$.

Les mots échangés occupent tous les moments : on négocie avant les batailles - des croisés sont envoyés à l'atabeg Kerbogha peu avant la bataille d'Antioche pour lui proposer de régler la question par un duel $^{25}$ - ou pendant les sièges - Balian d'Ibelin rencontre Saladin pour négocier la reddition de Jérusalem assiégée ${ }^{26}$. Le dialogue est le moyen principal de faire la paix : Anne Comnène, la fille de l'empereur Alexis Comnène, prend soin ainsi de préciser que son père fait envoyer des interprètes aux croisés «afin d'apaiser tous les conflits qui pourraient

\footnotetext{
${ }^{22}$ Aujourd'hui en Syrie du nord.

${ }^{23}$ Arthur S. Tritton et Hamilton A. R. Gibb « The first and second crusades from an anonymous syriac chronicle », Journal of the Royal Asiatic Society of Great Britain and Ireland, 1933, $\mathrm{n}^{\circ}$ 2, p. 273-305, cit. p. 279 : «They were noble natured, loved all men, and were good mediators at all time ».

${ }^{24}$ Daniel König, «Caught between cultures ? Bicultural personalities as crosscultural transmitters in the late antique and medieval Mediterranean », dans Acteurs des transferts culturels en Méditerranée médiévale, dir. Rania Abdellatif, Yassir Benhima, Daniel König, Elisabeth Ruchaud, München, Oldenbourg, coll. « Atelier des Deustchen Historischen Instituts Paris », 2012, p. 56-72. Daniel König souligne qu'il est difficile de se construire une identité stable lorsqu'on navigue sans cesse entre les cultures et entre les langues, ce qui peut se traduire par une «crise identitaire » plus ou moins grave.

${ }^{25}$ Anonyme Normand, Histoire anonyme de la première croisade [1924], trad. Louis Bréhier, Paris, Les Belles Lettres, coll. «Les Classiques de l'histoire de France au Moyen Âge », 2007, cit p. 149-151; Raymond d'Aguilers, Histoire des Francs qui prirent Jérusalem, Rennes, Les Perséides, coll. « Aux sources de l'histoire », 2006, cit p. 63.

${ }^{26}$ Abū Shāma, Le Livre des Deux Jardins. Histoire des deux règnes, celui de Nour ed-Dîn et celui de Salah ed-Dîn, dans RHC, Historiens Orientaux, tome IV, Paris, Imprimerie Nationale, 1898, p. 1-525, cit p. 328-329; Bahā ad-Dīn, Anecdotes et beaux traits de la vie du sultan Yûsuf, dans RHC, Historiens orientaux, tome III, Paris, Imprimerie Nationale, 1884, p. 3-370, cit p. 100.
} 
$\operatorname{surgir}^{27} \gg$. Faire la paix par les mots, en faisant circuler les mots : dans cette affirmation, on entend à la fois des échos du message christique, et la croyance sincère des médiévaux dans la performativité des paroles ${ }^{28}$. Plus généralement, l'importance de l'oralité dans les relations entre Latins, Grecs et musulmans atteste aussi de l'existence d'une identité nobiliaire qui unit toutes les religions, d'une koiné aristocratique ${ }^{29}$ dans laquelle l'éloquence, preuve d'intelligence, joue une place clé, aux côtés de la beauté et de l'habileté aux armes.

Échanger des mots est souvent un moyen de pallier l'impossibilité d'une rencontre réelle : Richard Cœur de Lion tente ainsi de rencontrer Saladin, mais celui-ci s'y refuse tant que la paix n'est pas signée ${ }^{30}$, et, finalement, les deux souverains ne se verront jamais face à face - malgré les nombreuses enluminures nous représentant leur duel, ou, plus tard, les réécritures romantiques d'un Walter $S \operatorname{cott}^{31}$.

\section{Sous les paroles}

Cet exemple nous amène à nous garder, là aussi, d'une vision trop apaisée, et trop apaisante. Les mots peuvent se charger de menaces, les paroles se faire insultes: pendant la troisième croisade, Hugues de Bourgogne fait chanter une «mauvaise chanson» sur Richard

${ }^{27}$ Anne Comnène, Alexiade, tome III, trad. Bernard Leib, Paris, Les Belles Lettres, coll. «Collection Byzantine», 1945, cit. livre X, chap. VI, 9, p. 209.

${ }^{28}$ Voir John L. Austin Quand dire, c'est faire, Paris, Éditions du Seuil, coll. «L'Ordre philosophique», 1970 ; et, pour une réflexion médiévale, Irène Rosier-Catach, La Parole efficace. Signe, rituel, sacré, Paris, Éditions du Seuil, coll. «Des Travaux », 2004.

${ }^{29}$ Johannes Preiser-Kapeller, « Heroes, traitors and horses. Mobile elite warriors in Byzantium and beyond, 500-1100 AD. », Draft text for a Presentation, Columbia University, 25 novembre 2013, en ligne sur Academia.edu, https://www.academia.edu/6436110/Heroes_traitors_and_horses._Mobile_elite_warri ors_in_Byzantium_and_beyond_500-1100_AD, page consultée le 22/11/2015.

${ }^{30}$ Bahā ad-Dīn, op. cit., p. 257 et p. 287.

${ }^{31}$ Pour un exemple d'enluminure, voir Londres, Royal Library, ms. Add. 42310, fol. 61. Walter Scott met en scène une telle rencontre dans son roman Le Talisman, publié pour la première fois en 1825 . 
d'Angleterre, pour l'humilier ${ }^{32}$. Le fait même d'échanger des mots peut être utilisé dans un but militaire: Renaud de Sidon profite ainsi de sa bonne connaissance de l'arabe et des relations d'amitié qu'il a liées avec Saladin pour faire traîner les négociations, donnant le temps à ses chevaliers de fortifier son château ${ }^{33}$; et Bohémond de Tarente, près d'un siècle auparavant, noue des relations avec l'un des habitants d'Antioche pour le pousser à lui livrer la ville ${ }^{34}$. N'allons pas non plus imaginer que les dialogues débouchent systématiquement sur la conclusion de la paix : selon Guillaume de Tyr, les Templiers tuent, en 1173, un émissaire du Vieux de la Montagne, chef des Assassins, qui avait offert au roi de Jérusalem de se convertir à la foi chrétienne ${ }^{35}$ - ce qui aurait fait perdre aux Templiers l'important tribut que leur versent les Nizârites, raison pour laquelle l'ordre militaire préfère dès lors mettre fin à la négociation en assassinant l'assassin. Porteurs de lettres, les messagers se font les vivantes incarnations du dialogue, en sorte qu'en tuer un, c'est clore le dialogue, mettre un terme à l'échange de mots, la mort du messager jouant comme le point final du message.

Enfin, le rôle même des mots peut être critiqué. En effet, ceux-ci n'occupent pas forcément une place similaire dans les codes politiques des différents acteurs. Un exemple est célèbre : en 1108, Tancrède, régent d'Antioche, allié à Rodwân, émir d'Alep, livre bataille contre Djawâli de Mossoul, allié à Josselin d'Édesse. Avant la bataille, les deux seigneurs

\footnotetext{
${ }^{32}$ Ambroise, L'Estoire de la Guerre Sainte. Histoire en vers de la troisième croisade (1190-1192), trad. Gaston Paris, Paris, Imprimerie Nationale, 1897, cit. p. 285, v. 10653-10658.

${ }^{33}$ Bahā ad-Dīn, op. cit., p. 121-129.

${ }^{34}$ Épisode que l'on trouve dans toutes les chroniques de la première croisade. Voir, sur ce personnage, l'analyse percutante de Robert Levine, «The Pious traitor: rhetorical reinventions of the fall of Antioch », Mittellateinisches Jahrbuch, 1998, vol. $33, \mathrm{n}^{\mathrm{o}} 1$, p. 59-80.

${ }^{35}$ Guillaume de Tyr, Historia rerum in partibus transmarinis gestarum, dans RHC, Historiens Occidentaux, tome I, Paris, Imprimerie Royale, 1844, p. 1-1156, cit. livre XX, chap. 30, p. 998.
} 
francs s'avancent entre les rangs et prennent le temps de parler, ce qui plonge les deux seigneurs musulmans dans des affres d'angoisse. En effet, ceux-ci craignent une trahison, ne sachant pas que le discours avant la bataille fait partie des pratiques latines ${ }^{36}$. De la même manière, Anne Comnène, la fille de l'empereur Alexis, peut écrire que les Francs «sont de grands bavards ${ }^{37} »$. Quelques années plus tard, Eudes de Deuil insiste sur le fait que les Grecs sont verbeux, produisant de longs discours creux, tissés de paroles douces et flatteuses mais vides de $\operatorname{sens}^{38}$. Le reproche, par sa symétrie, renvoie au rôle différent de la parole dans ces deux cultures : chez les Latins, tous les nobles doivent pouvoir parler, au point que l'une des punitions du chevalier coupable de trahison est précisément de le priver de son droit à la parole : il n'a plus voix à rien, dit par exemple le Livre au Roi ${ }^{39}$. Chez les Grecs, le discours doit être ciselé, savamment construit, en recourant volontiers à l'hyperbole ou à des métaphores complexes, dans l'héritage de la rhétorique antique. Pour les Grecs, trop de Latins parlent, et pour les Latins, les Grecs parlent trop. Les mots qui s'échangent tournent ici à vide, creusant de plus en plus les

\footnotetext{
${ }^{36}$ Ibn abī Tayyi', cité par Ibn Furāt, Ta'rīkh al-duwal wa l-mulūk, ms. Vienne, AF 117, vol. 1, fol. $24 v^{\circ}-25 v^{\circ}$, cité et traduit par Claude Cahen, Orient et Occident au temps des Croisades, Paris, Aubier Montaigne, coll. «Collection historique », 1983, p. 226. Voir, sur le même événement, Claude Cahen, «Un Épisode épico-féodal franc dans une chronique arabe », dans La Noblesse au Moyen Âge. Essais à la mémoire de Robert Boutruche, dir. Philippe Contamine, Paris, Presses Universitaires de France, 1976, p. 129-132 ; Anne-Marie Eddé, «L'Honneur des chevaliers francs dans les sources arabes à l'époque des croisades », dans L'Islam au carrefour des civilisations médiévales, dir. Michel Sot et Dominique Barthélémy, Paris, Presses Universitaires de Paris-Sorbonne, coll. «Cultures et civilisations médiévales », 2012, p. 135-153.

${ }^{37}$ Anne Comnène, Alexiade, op. cit., livre X, chapitre IX, 4, p. 221.

${ }^{38}$ Eudes de Deuil, La Croisade de Louis VII, op. cit., II, p. 29, et III, p. 43. C'est, dans une certaine mesure, un topos hérité de l'Antiquité, comme le souligne Marc Carrier, L'Autre chrétien pendant les croisades. Les Byzantins vus par les chroniqueurs du monde latin (1096-1261), Saarbrücken, Éditions universitaires européennes, 2012, en particulier p. 68-95.

${ }^{39}$ Le Livre au Roi, op. cit., chap. 46, p. 273.
} 
fossés puisque renvoyant les acteurs à leurs différences. On peut donc souhaiter échanger plus que des mots, d'où l'importance des objets.

\section{Des cadeaux}

\section{Faire lien}

Comme pour la parole, l'importance des dons et des cadeaux recoupe le rôle majeur de la largesse dans les sociabilités aristocratiques et dans l'idéologie chevaleresque : être noble, c'est donner - au sens propre, puisque le terme même de lord veut dire, étymologiquement, «donneur de pain ${ }^{40} »$. De nombreux travaux, inspirés par les réflexions de Marcel Mauss ${ }^{41}$, ont souligné à quel point le don et le contredon étaient créateurs de lien social au Moyen $\hat{A}_{\mathrm{ge}}{ }^{42}$ : on donne pour se faire des amis, ou pour faire la paix ${ }^{43}$. Il n'est dès lors pas surprenant que l'on retrouve ces pratiques dans les rencontres et les contacts entre communautés, et ce dans tout le Proche-Orient médiéval ${ }^{44}$. Lorsque Godefroy de Bouillon est sollicité par l'émir d'Hasarth, Omar, qui désire

40 Marc Bloch, La Société féodale [1939-1340], Paris, Albin Michel, coll. «L'évolution de l'humanité », 1969, p. 259.

${ }^{41}$ Marcel Mauss, Essai sur le don : forme et raison de l'échange dans les sociétés archaïques [1923], Paris, Presses Universitaires de France, coll. «Quadrige », 2012 (1923).

${ }^{42}$ Stephen D. White, «Service for fiefs or fiefs for service. The politics of reciprocity », dans Negotiating the gift. Pre-modern figurations of exchange, Göttingen, dir. Gadi Algazi, Valentin Groebner et Bernhard Jussen, Vandenhoeck \& Ruprecht, 2003, p. 63-98. Voir aussi Eliana Magnani, «Les Médiévistes et le don. Avant et après la théorie maussienne », Bulletin du centre d'études médiévales d'Auxerre (BUCEMA), 2008, Hors-série $\mathrm{n}^{\circ} 2$, en ligne.

${ }^{43}$ Voir par exemple les échanges de cadeaux après la conclusion de la paix entre Renaud de Châtillon, prince d'Antioche, Thoros d'Arménie et le basileus Manuel Comnène, tels qu'ils sont décrits par Grégoire le Prêtre, Chronique, dans RHC, Documents Arméniens, I, Paris, Imprimerie Nationale, 1869, p. 151-201, cit. p. 187. Sur le rôle des cadeaux pour faire la paix, voir Yvonne Friedman, "Peacemaking : perceptions and practices in the medieval Latin East», dans The Crusades and the Near East, dir. Conor Kostick, New York, Routledge, 2011, p. 229 257, en particulier p. 244-246.

${ }^{44}$ Anthony Cutler, «Gift and gift exchange as aspects of byzantine, arab, and related economies », Dumbarton Oaks Papers, 2001, n 55, p. 247-278. 
devenir son vassal - vocabulaire occidental, et on ignore quel degré de sujétion politique Omar avait réellement en tête - et lui envoie donc des otages, dont son propre fils, Godefroy lui fait envoyer en retour des objets précieux : «le duc, d'après le conseil de ceux qui l'entouraient, donna à son nouvel allié un casque parfaitement bien plaqué en or et en argent, et une cuirasse d'une grande beautét ${ }^{45} \gg$. Le don est triplement symbolique. D'abord, la préciosité des objets sert à mettre en scène la grandeur de Godefroy, image du seigneur généreux. La nature des objets participe aussi de la logique de l'échange : en donnant des armes, le duc de BasseLotharingie accomplit son rôle de seigneur, offrant à son homme le nécessaire pour en recevoir l'auxilium. Enfin, par le fait même de donner, Godefroy transforme un étranger, a priori hostile, en familier - d'autant plus que ces armes, nous précise le chroniqueur, appartiennent à l'un des chevaliers de Bouillon qui font partie de la mesnie du duc. En faisant circuler les armes - d'un chevalier au duc et du duc à un nouveau vassal le texte souligne la possibilité d'intégrer un musulman dans le cercle de confiance du chef chrétien: quelques pages plus loin, Albert d'Aix emploie significativement le terme de fidei, fidèle, avec ses connotations à la fois politiques et religieuses, pour parler d'Omar.

Le fait d'offrir recoupe, là aussi, cette koiné aristocratique faite de beaux gestes, d'honneur, de panache et d'orgueil. Saladin offre ainsi deux chevaux à Richard, son adversaire, au beau milieu d'une bataille ${ }^{46}$; on le voit également laisser les Francs quitter Jérusalem avec leurs trésors,

\footnotetext{
45 Albert d'Aix, Liber christianae expeditionis pro ereptione, emundatione, restitutione sanctae Hierosolymitanae ecclesiae, dans RHC, Historiens occidentaux, tome IV, Paris, Imprimerie Nationale, 1879, p. 265-713, cit. livre V, chap. 12, p. 439 : «Dux ex consilio suorum viro sibi confoederato galeam, auro et argento mire insertam, et loricam magnae honestatis contulit. ». Je traduis.

${ }^{46}$ L'Estoire de Eracles empereur et la conqueste de la terre d'outremer, dans RHC, Historiens occidentaux, tome II, Paris, Imprimerie impériale, 1859, cit. livre XXVI, chap. 15, p. 197.
} 
malgré les reproches de son entourage ${ }^{47}$. Cette largesse du sultan, célébrée par toutes les sources, résultat d'une habile mise en scène et d'une propagande savamment orchestrée ${ }^{48}$, explique en partie la transformation de Saladin en héros chevaleresque dans la littérature occidentale $^{49}$.

\section{Pièges et poisons}

Attention à ne pas imaginer une société apaisée, dans laquelle tout le monde se ferait des cadeaux somptueux avec empressement. Comme pour les paroles, la circulation des objets se double de tensions, renforcées par les oppositions politiques et religieuses. Les objets échangés peuvent être profondément ambivalents: Yvonne Friedman relève ainsi l'importance du faucon, échangé entre Francs et musulmans Usāma ibn Munqidh en offre un bel exemple ${ }^{50}$ - et note que c'est un beau symbole de la paix, car la chasse est le divertissement nobiliaire par excellence $^{51}$. En s'échangeant des faucons, les Francs et les musulmans s'appuient sur les codes culturels qu'ils ont en commun, sur cette koiné aristo-chevaleresque qui les unit, et l'oiseau de proie joue comme une mise en abyme de la paix créée par le don. L'analyse est pertinente, mais on peut aussi relever que le faucon reste un oiseau de proie, et que la chasse s'apparente de fort près à la guerre - en particulier chez Usāma ibn

${ }^{47}$ 'Imād ad-Dīn al-Isfahān̄̄, Conquête de la Syrie et de la Palestine par Saladin (Al-Fath al qussi fî l-fath al-qudsî), trad. Henri Massé, Paris, P. Geuthner, coll. « Documents relatifs à l'histoire des croisades », 1972, cit. p. 61-62.

48 Anne-Marie Eddé, Saladin, Paris, Flammarion, 2008, en particulier p. 176-200.

${ }^{49}$ Ibid.; Jean Richard, «Les Transformations de l'image de Saladin dans les sources occidentales », Revue des mondes musulmans et de la Méditerranée, 2000, $\mathrm{n}^{\circ}$ 89-90, en ligne.

${ }^{50}$ Usāma ibn Munqidh, Des enseignements de la vie. Souvenirs d'un gentilhomme syrien du temps des Croisades, trad. André Miquel, Paris, Imprimerie Nationale, coll. « Collection orientale de l'imprimerie nationale », 1983, cit. p. 391.

${ }^{51}$ Yvonne Friedman, «Peacemaking: perceptions and practices in the medieval Latin East », art. cit. 
Munqidh, qui file la métaphore de la chasse sur toute son œuvre ${ }^{52}$, mais aussi chez les auteurs occidentaux, prompts à comparer les guerriers à des faucons ${ }^{53}$. S'échanger des faucons, c'est donc aussi, au moins métaphoriquement, situer l'échange dans le domaine de l'affrontement. En effet, tous les familiers du potlatch le savent bien, l'échange touche de près à la rivalité ${ }^{54}$. Philippe Haugeard a ainsi souligné que la largesse était toujours un moyen de domination ${ }^{55}$ : les acteurs médiévaux en ont bien conscience, et recevoir, a fortiori recevoir des mains d'un adversaire, est toujours suspect. Ainsi, lorsque Richard Cœur de Lion souhaite envoyer un cadeau à Saladin, il demande d'abord la permission - acte en soi intéressant, qui souligne à la fois la prudence du roi d'Angleterre et la profonde ambivalence des cadeaux - et on lui répond: «cette autorisation lui est donnée à la condition qu'il recevra l'équivalent de ce qu'il offrira $^{56} \gg$. Comment mieux dire que la triple obligation maussienne est une réalité, mais une réalité qui a sans cesse besoin d'être réaffirmé et renégociée ? Comment mieux souligner que, derrière les cadeaux, sous le

\footnotetext{
${ }^{52}$ Paul M. Cobb, «Infidel dogs. Hunting crusaders with Usâma ibn Munqidh », Crusades, 2007, $\mathrm{n}^{\mathrm{o}}$ 6, p. 57-68.

${ }^{53}$ Voir par exemple Bertrand de Born qui écrit que Richard Cœur de Lion « ans vol guerra mais que calh' esparviers », «veut la guerre plus que l'épervier la caille », trad. Paul Fabre, Anthologie des troubadours, Paris, coll. «Medievalia», 2011, cit. p. 188-189. Même métaphore pour Tancrède chez Raoul de Caen, Gesta Tancredi in Expedition Hierosolymitana, dans RHC, Historiens Occidentaux, tome III, Paris, Imprimerie impériale, 1866, p. 587-716, cit chap. CXXXIX, p. 703, et chap. CLVI, p. 715.

${ }^{54}$ Rappelons que le potlatch est un rituel basé sur le don, pratiqué originellement dans le monde amérindien et océanien, très étudié par les anthropologues, dans lequel chacun cherche à surpasser les autres par les dons qu'il offre, afin de conforter sa position sociale. Le potlatch comporte généralement, sinon systématiquement, un aspect de gaspillage ostentatoire : on brûle ou on jette les cadeaux, pour montrer que le don lui-même compte plus que l'objet donné. Voir Isabelle Schulte-Tenckhoff, Potlatch, conquête et invention : réflexion sur un concept anthropologique, Lausanne, Éditions d'en bas, coll. «Le Forum Anthropologique », 1986.

${ }_{55}$ Philippe Haugeard, Ruses médiévales de la générosité. Donner, dépenser, dominer dans la littérature épique et romanesque des XII et XIII siècles, Paris, Honoré Champion, coll. « Nouvelle bibliothèque du Moyen Âge », 2013.

${ }^{56}$ Bahā ad-Dīn, op. cit., p. 228
} 
voile de la largesse, se cachent des enjeux politiques majeurs? Recevoir plus que l'on ne donne, c'est recevoir trop, car c'est devenir le débiteur de l'autre - position sociale d'infériorité impossible pour Saladin, dont Bahā ad-dīn nous dit que «personne ne pouvait le surpasser quand il s'agissait de cadeaux ${ }^{57} »$. Si les cadeaux sont bien mis au service de la fabrication du lien social, on voit que ce lien ne cesse d'être agonistique.

Plus encore, comme pour les mots, le fait même d'offrir ou de recevoir peut être objet de critiques. On voit ainsi Henri de Champagne demander à Saladin de lui offrir une robe d'honneur - c'est la khil'a, ou tashrif, objet occupant une place importante dans la grammaire politique $\operatorname{arabe}^{58}$. Henri précise qu'il la portera, par respect pour Saladin, même si cela est mal vu dans son camp ${ }^{59}$. On peut penser, bien sûr, que Henri ne soulève ces réticences que pour mieux se mettre en valeur dans son rôle d'intermédiaire modéré; mais l'argument peut aussi être fondé, et renvoyer à l'opinion commune parmi les croisés. On sait que Richard fut critiqué pour avoir accepté des cadeaux - des chameaux et des tentes - de la part du frère de Saladin :

Car Saffadin tant le déçut

Que li reis ses presenz reçut.

Messagier vindrent e alerent

Qui les presenz al rei porterent.

Dont il fud blasmé durement

Et en parla on malement ${ }^{60}$.

${ }^{57}$ Id, p. 320.

${ }^{58}$ Gavin R. G Hambly, «From Baghdad to Bukhara, from Ghazna to Delhi : the khil'a ceremony in the transmission of kingly pomp and circumstance », dans Robes and honor: The medieval world of investiture, dir. Stewart Gordon, Houndmills : Palgrave, coll. «The New Middle Ages », 2001, p. 95-135.

${ }^{59}$ Ibn al-Athīr, Al-Kāmil fì al-tārīkh, trad. Francesco Gabrieli, Chroniques arabes des Croisades, Arles, Sindbad-Actes Sud, coll. «La bibliothèque arabe », 2014, cit. p. 267.

${ }^{60}$ Ambroise, op. cit., vers 7405-7410, p. 198. 
Pour ces critiques, le contact devient ici compromis et le compromis corruption - au sens pur, l'âme impeccable du croisé se tachant au contact des païens comme un métal rouille au contact de l'eau. Ce qui est critiqué ici, c'est l'idée même d'une circulation. Le cadeau signale ainsi une ouverture vers l'autre jugée au mieux suspecte, au pire condamnable: en 1130, Alice d'Antioche est accusée d'avoir cherché à s'allier aux musulmans, tentative marquée par l'offrande d'un cheval blanc à Zengui ${ }^{61}$. Le cadeau est intercepté par l'armée royale, et, faute de pouvoir punir sa propre fille, le roi Baudouin fait mettre à mort le messager, preuve que le prix à payer pour ces intermédiaires n'est pas uniquement symbolique.

Offrir, c'est donc toujours, au moins en partie, s'offrir, que l'on donne ou que l'on reçoive. En faisant rimer « reçut» avec «déçut», Ambroise, dans le passage cité plus haut, met en scène et en son une circulation trahie, puisque Saladin offre des cadeaux sans vouloir vraiment conclure la paix. Dès lors qu'elle n'est plus sincère, l'offrande se fait achat: le Ménestrel de Reims signale ainsi que Saladin utilise sa richesse pour acheter des barons traitres à leur roi ${ }^{62}$. Tout comme les mots, arrachés à leur usage créateur dès lors qu'ils sont utilisés pour gagner du temps ou pour insulter l'autre, la générosité change ici de visage, la largesse devenant suspecte et corruptrice. Les sources sont nombreuses à exprimer une profonde méfiance des cadeaux de l'autre : Eudes de Deuil comme Guillaume de Tyr reprennent le fameux timeo

\footnotetext{
${ }^{61}$ Guillaume de Tyr, op. cit., livre XIII, chap. 27, p. 600. Sur Alice et son action, voir Thomas S. Asbridge, «Alice of Antioch : a Case Study of Female Power in the Twelfth Century », dans The Experience of Crusading, II. Defining the Crusader Kingdom, dir. Peter Edbury et Jonathan Philips, Cambridge, Cambridge University Press, 2003, p. 29-47.

${ }^{62}$ Récits d'un Ménestrel de Reims, éd Natalis de Wailly, Paris, Renouart, 1876, chap. VI, § 33-35, p. 48.
} 
danaos et dona ferentes ${ }^{63}$, et Guibert de Nogent s'empresse de noter que Godefroy, trop confiant dans l'amitié des musulmans, est empoisonné par des fruits qu'ils lui font envoyer ${ }^{64}$ - une belle image, qui évoque évidemment l'analogie gift - gift, cadeau-poison ${ }^{65}$. Sans aller jusqu'au poison, on voit que les cadeaux sont donc un piège: dans une autre version de l'anecdote citée plus haut, Saladin offre un cheval à Richard... mais un cheval dressé pour capturer le roi et le ramener prisonnier au camp musulman, ce que déjoue Richard en ne montant pas sur l'animal ${ }^{66}$. Le récit est ici limpide : en acceptant les cadeaux de l'autre, on risque sa liberté, et donc sa vie.

Guibert de Nogent rapporte la mort, sous les coups des musulmans, d'un chevalier parfait, qu'il connaissait personnellement, nommé Matthieu, et précise que cette mort est en adéquation avec son nom, qui veut dire «don de Dieu ${ }^{67} »$ : pour le moine, écrivant sa chronique dans son monastère français, loin de l'Orient, les infidèles sont par excellence ceux qui arrachent, ceux qui, ennemis à la fois du Christ, des chrétiens, et de la chrétienté, volent les dons de Dieu. Au contraire, les sources locales, produites dans le terreau de cet Orient latin, mettent en scène l'importance des échanges, de mots et de cadeaux, dans la mise en place de rapports sociaux qui, bien plus que de franchir les barrières confessionnelles, les redessinent en profondeur. Si l'on gagne ainsi à

\footnotetext{
${ }^{63}$ Eudes de Deuil, op. cit., II, p. 29 ; Guillaume de Tyr, op. cit., livre XI, chap. 6, p. 461. Voir l'analyse de Marc Carrier, L'Autre chrétien pendant les croisades. Les Byzantins vus par les chroniqueurs du monde latin (1096-1261), op. cit., p. 156-164.

${ }^{64}$ Guibert de Nogent, Gesta Dei per Francos, dans RHC, Historiens occidentaux, tome IV, Paris, Imprimerie Nationale, 1879, p. 113-263, cit. livre VII, chap. 25, p. 245 .

${ }^{65}$ Conceptualisée et théorisée par Marcel Mauss, «Gift, gift », dans Mélanges offerts à Charles Andler, Strasbourg, Imprimerie Alsacienne, 1924, p. 243-247. Voir Erhard Schüttpelz, «Gift, gift - La terminologie du don chez Marcel Mauss », Trivium, 2014, $\mathrm{n}^{\mathrm{o}} 17$, en ligne.

${ }^{66}$ Chronique d'Ernoul et de Bernard le Trésorier, Paris, Renouard, 1871, p. 281282.

${ }^{67}$ Guibert de Nogent, op. cit, livre IV, chap. 18, p. 184.
} 
sortir du schéma de l'arrachement, il ne faut pas non plus tomber dans le mythe de l'union. Car tout ne circule pas: les femmes, par exemple, objets d'échange dans le cadre de stratégies matrimoniales ${ }^{68}$, ne circulent que très peu entre les communautés - que l'on pense à Richard Cœur de Lion offrant sa sœur en mariage au frère de Saladin, pratique habituelle en Occident pour faire la paix, mais qui se heurte ici à l'opposition du clergé, des nobles... et de Jeanne d'Angleterre elle-même ${ }^{69}$. Lorsqu'il évoque cette tentative inaboutie, le texte d'Imâd ad-Dîn se teinte de l'amertume des occasions manquées: «dans les conditions présentes, quel moyen d'aboutir à l'amitié, alors que la diversité de religion nous impose l'hostilité $?^{70} »$. Conclusion pessimiste, tissée de la lassitude de l'affrontement, mais extrêmement logique pour une époque qui fait toujours de la religion le ciment de l'appartenance sociale, autrement dit le nœud de l'identité à la fois individuelle et collective. Dans ce contexte, l'amitié passe nécessairement par la conversion, seule à même de faire disparaître l'altérité ; la paix politique et sociale ne se conçoit donc, ultimement, que dans une société mono-confessionnelle ${ }^{71}$.

Les résultats, au terme de cette trop courte analyse, sont mitigés. On a souligné l'importance de la circulation, dans les relations politiques et diplomatiques entre communautés, des mots et des objets ${ }^{72}$. Cet échange ne sert pas à nier la différence, mais plutôt à réduire la distance pour permettre ensuite la création d'un lien plus durable: conclusion

${ }^{68}$ Georges Duby, Le Chevalier, la femme, le prêtre. Le mariage dans la France féodale, Paris, Hachette, coll. «Pluriel », 1997.

${ }^{69}$ Bahā ad-Dīn, op. cit, p. 278.

70 'Imād ad-Dīn al-Isfahānī, op. cit., p. 351.

71 Jonathan Riley-Smith, "Peace never established : the case of the Kingdom of Jerusalem », Transactions of the Royal Historical Society, 1978, $\mathrm{n}^{\circ}$ 28, p. 87-102.

${ }^{72}$ Cette importance participe aussi de la circulation du pouvoir dans la société féodale occidentale. Voir Florian Besson, «La Justice, le souverain et le pouvoir dans le Livre au Roi : de l'arbre au rhizome », dans Autour des Assises de Jérusalem, dir. Jérôme Devard et Bernard Ribémont, à paraître. 
d'une paix, mise en place d'une alliance, naissance d'une amitié. Il s'agit de faire circuler, d'échanger, sous la forme de la triple obligation maussienne du donner-recevoir-rendre, non pas pour se dire identique, mais, simplement, pour vivre avec. Plus encore qu'un pont tendu vers l'autre, le don fonctionne comme une porte que l'on ouvre - prudemment, et sans être jamais à l'abri qu'un courant d'air la referme brutalement. La violence, sans cesse, s'immisce au cœur du cercle, qu'elle se cache derrière l'objet échangé, qu'elle torde le sens des paroles prononcées ou qu'elle introduise un rapport de domination là même où on le croyait conjuré.

L'échange reste à la fois nécessaire et dangereux - profondément ambivalent. Et cette ambiguïté permet de renvoyer dos à dos deux mythes contemporains, ancrés au cœur du libéralisme, celui d'un choc des civilisations, et celui, plus insidieux puisque plus séduisant, d'une pacification définitive du monde par l'échange. Ce que nous dit le Proche-Orient médiéval, en somme, c'est que, pour faire lien et rencontrer l'autre, l'échange est nécessaire, mais jamais suffisant. 